This item was submitted to Loughborough's Institutional Repository (https://dspace.lboro.ac.uk/) by the author and is made available under the following Creative Commons Licence conditions.

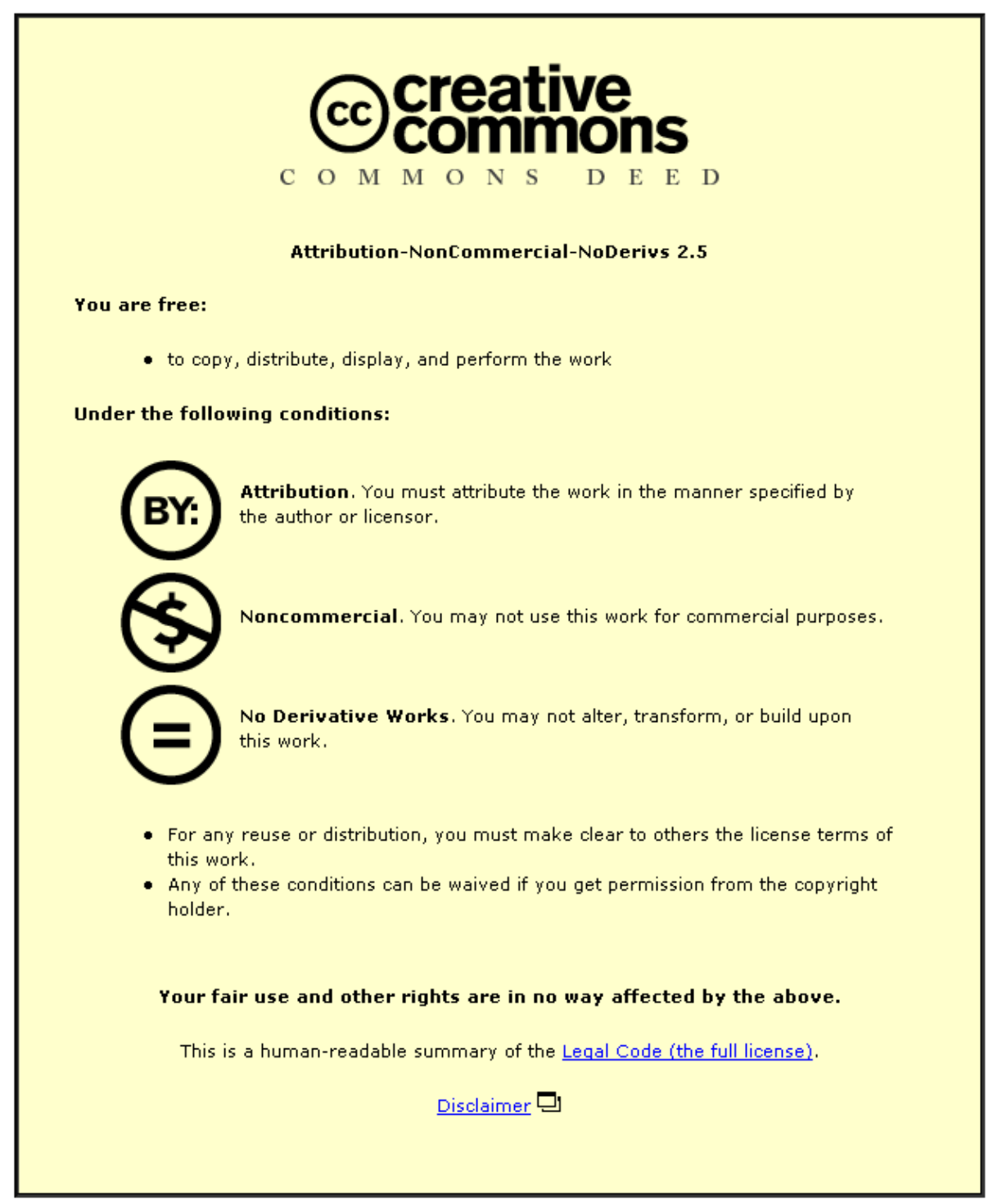

For the full text of this licence, please go to: http://creativecommons.org/licenses/by-nc-nd/2.5/ 


\section{JOINT TRANSMITTER AND RECEIVER DESIGN FOR MIMO CHANNEL SHORTENING}

\author{
Cenk Toker and Sangarapillai Lambotharan
}

\author{
CDSPR, Dept. of Electronic Engineering \\ King's College London, WC2R 2LS, UK \\ cenk.toker@ieee.org, s.lambotharan@kcl.ac.uk
}

\author{
Jonathon A. Chambers \\ Cardiff School of Engineering, \\ Cardiff University, Cardiff CF24 OYF UK \\ chambersj@cf.ac.uk
}

\begin{abstract}
The problem of joint transmitter and receiver design for multi-input multi-output (MIMO) channel shortening for frequency-selective fading channel is addressed. A frequency domain approach is followed which is equivalent to infinite length time-domain channel shortening equalizers (TEQ). A practical joint space and frequency waterfilling algorithm is also provided for optimum transmit power loading. It is demonstrated that the finite length TEQ suffers from a flooring effect on the compression ratio performance, whereas the proposed method overcomes this disadvantage. The noise amplification and the compression performance of the proposed joint tranceiver method is found to be better than both finite and infinite length receiver-only designs, with a gain of order of $3 \mathrm{~dB}$ for a $2 \times 2 \mathrm{MIMO}$ channel.
\end{abstract}

\section{INTRODUCTION}

Maximum-likelihood sequence estimation (MLSE) and multicarrier modulation (MCM) are effective tools to mitigate frequencyselective fading channels. However, the computational complexity of MLSE grows exponentially with the multipath spread of the channel. Moreover, in MCM, a cyclic prefix of comparable length with the multipath spread has to be appended to the transmission frame. Clearly, the length of the multipath spread either causes an increase in the complexity of the receiver or a decrease in the throughput of the system. In order to avoid these disadvantages, the channel should be shortened to a desired length by the aid of a channel shortening filter. When compared to the complete equalization of the channel, channel shortening filter provides better performance due to the extra degree of freedom it possesses.

Channel shortening filter design has been a hot research topic since 1970's, starting with [1]. Although there have been many designs satisfying different criteria [2], the minimum-mean square error (MMSE) criterion has been the most widely investigated one. In this paper, we will address the channel shortening problem for multi-input multi-output (MIMO) channels under the MMSE criterion with the orthogonality constraint [3] imposed on the target impulse response (TIR).

A joint transmitter and receiver filter design approach for channel shortening is provided. Even though joint transmitter receiver design exists for equalization, to our best knowledge, it is the first time this is addressed for a channel shortening filter design. If the transmitter has the channel state information (Tx-CSI), the proposed algorithm may prove to be useful since it provides much better performance compared to a receiver-only design. For example, in wireline systems, such as xDSL, the rate of change of the channel is very slow and it is possible for the transmitter to get CSI.

A frequency-domain approach similar to [4], [5] is adopted and the resulting design is equivalent to infinite length time-domain equalizer (TEQ). Under certain transformations, it will be demonstrated that the MIMO system can be decoupled into a set of independent single-input single-output (SISO) subsystems, greatly simplifying the design procedure. The optimization results in a space and frequency waterfilling algorithm which enhances the performance. We will provide a practical waterfilling algorithm which finds the optimum setting in a finite number of iterations, in contrast to [4] where this can be infinite.

A function in the time domain will be denoted by $f(k)$ and its frequency response by $f(\omega)$. A vector function will be represented by small boldface, $\mathbf{f}(\omega)$, whereas a matrix function will be denoted by capital boldface, $\mathbf{F}(\omega)$.

\section{PROBLEM STATEMENT}

Consider the block diagram given in Figure 1. It corresponds to the joint transmitter and receiver design problem of MIMO channel shortening based on the MMSE criterion. The blocks in the upper branch correspond to the transmitter filter, $\mathbf{C}(\omega)$, the channel, $\mathbf{H}(\omega)$, and the receiver filter, $\mathbf{Q}(\omega)$, all in their frequency response representations. The number of transmit antennas is assumed $n_{T}$ whereas the number of receive antennas is $n_{R}$. It is assumed that the number of input data streams is $n_{i}$. Therefore the dimensions of $\mathbf{C}(\omega), \mathbf{H}(\omega)$, and $\mathbf{Q}(\omega)$ are $n_{T} \times n_{i}, n_{R} \times n_{T}$, and $n_{i} \times n_{R}$, respectively. The data $\mathbf{d}(k)$ and the noise $\mathbf{n}(k)$ have power spectral density matrices $\boldsymbol{\Phi}_{d d}(\omega)$ and $\boldsymbol{\Phi}_{n n}(\omega)$ with dimensions $n_{i} \times n_{i}$ and $n_{R} \times n_{R}$, respectively.

Although the signals and filters on the upper branch exist physically, the lower branch is a virtual one representing the desired model and the upper branch is aimed to approximate the lower one. The $n_{i} \times n_{i}$ filter $\mathbf{B}(\omega)$ is named as the target impulse response (TIR) and it represents the target system model which is the convolution of the transmitter and receiver filters and the channel. The ultimate goal is to shorten the original channel of length $n_{H}$ to an equalized one with much shorter length, $n_{B}$, i.e. $n_{B}<n_{H}$. Hence, the TIR can be written in the z-domain as follows

$$
\mathbf{B}\left(z^{-1}\right)=\mathbf{B}_{0}+\mathbf{B}_{1} z^{-1}+\cdots+\mathbf{B}_{n_{B}-1} z^{-\left(n_{B}-1\right)}
$$

where $\mathbf{B}_{k}, k=0, \ldots, n_{B}-1$ are the $n_{i} \times n_{i}$ matrix taps of the TIR at the corresponding delay. The term $e^{-j \omega m} \mathbf{I}$ accounts for the 


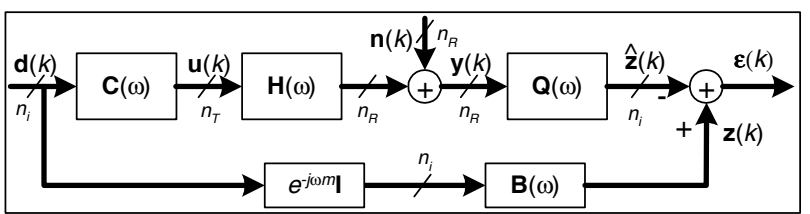

Fig. 1. The structure of the channel shortening problem of a MIMO frequency selective fading channel, $\mathbf{H}(\omega)$, where the transmitter filter $\mathbf{C}(\omega)$, the receiver filter $\mathbf{Q}(\omega)$ and the target impulse response $\mathbf{B}(\omega)$ are jointly optimised to minimise the mean square error criterion depending on the error signal $\varepsilon(k)$.

delay which is an important parameter in FIR filter design as it is influencing the performance [3]. However, it will be demonstrated later that the present design is insensitive to this delay.

The $n_{i} \times 1$ error signal $\varepsilon(k)$ is defined as the difference between the desired signal $\mathbf{z}(k)$ at the output of the TIR, and the equalized signal $\hat{\mathbf{z}}(k)$ at the output of the receiver filter as follows

$$
\begin{aligned}
& \varepsilon(k)=\mathbf{z}(k)-\hat{\mathbf{z}}(k) \\
& =\left[q^{-m} \mathbf{B}(k)-\mathbf{Q}(k) * \mathbf{H}(k) * \mathbf{C}(k)\right] * \mathbf{d}(k)-\mathbf{Q}(k) * \mathbf{n}(k)
\end{aligned}
$$

where $q^{-1}$ is the unit delay operator and '*' denotes the convolution operator. Our goal is to minimize the mean square error

$$
J(\mathbf{Q}, \mathbf{C}, \mathbf{B})=\operatorname{tr} E\left\{\varepsilon(k) \varepsilon^{H}(k)\right\} .
$$

Substituting (3) into (4) and applying Parseval's formula, we obtain (5) on the top of the next page.

Without any constraints, the optimization will converge to the trivial solution of $\mathbf{B}=\mathbf{Q}=\mathbf{0}$ and $\mathbf{C}=\infty$ which is meaningless. Therefore, we impose the limited transmit power constraint on $\mathbf{C}$ as

$$
\operatorname{tr} \frac{1}{2 \pi} \int_{-\pi}^{\pi} \mathbf{C}(\omega) \Phi_{d d}(\omega) \mathbf{C}^{H}(\omega) d \omega \leq P_{o}
$$

and the orthogonality constraint on $\mathbf{B}$ as

$$
\frac{1}{2 \pi} \int_{-\pi}^{\pi} \mathbf{U}^{H}(\omega) \mathbf{B}(\omega) \mathbf{B}^{H}(\omega) \mathbf{U}(\omega) d \omega=\mathbf{I}
$$

for some unitary transformation $\mathbf{U}(\omega)$ which will be explained shortly. In the sequel, the parenthesis $(\omega)$ will be dropped where convenient.

\section{RECEIVER FILTER DESIGN}

The optimum receiver filter $\mathbf{Q}_{\text {opt }}(\omega)$ can be found by setting the gradient of $J$ in (5) with respect to $\mathbf{Q}(\omega)$ to zero

$$
\mathbf{Q}_{o p t}=e^{-j m \omega} \mathbf{B} \boldsymbol{\Phi}_{d d} \mathbf{C}^{H} \mathbf{H}^{H}\left(\mathbf{H C} \boldsymbol{\Phi}_{d d} \mathbf{C}^{H} \mathbf{H}^{H}+\boldsymbol{\Phi}_{n n}\right)^{-1} .
$$

Substitution of (8) into (5) yields

$J(\mathbf{C}, \mathbf{B})=\operatorname{tr} \frac{1}{2 \pi} \int_{-\pi}^{\pi}\left\{\mathbf{B}\left[\boldsymbol{\Phi}_{d d}^{-1}+\mathbf{C}^{H} \mathbf{H}^{H} \boldsymbol{\Phi}_{n n}^{-1} \mathbf{H C}\right]^{-1} \mathbf{B}^{H}\right\} d \omega$.

\section{DIAGONALIZATION}

Minimization of (9) with respect to $\mathbf{C}(\omega)$ and $\mathbf{B}(\omega)$ is not straightforward due to the matrix structure involved and the constraints in (6) and (7). The design procedure is greatly simplified by casting the problem into a set of independent single-input single-output (SISO) subsystems, through certain transformations as follows.

Consider the eigendecomposition of the spectral density of the input $\mathbf{d}(k), \boldsymbol{\Phi}_{d d}(\omega)$ as

$$
\boldsymbol{\Phi}_{d d}(\omega)=\mathbf{U}(\omega) \mathbf{K}_{d d}(\omega) \mathbf{U}^{H}(\omega)
$$

which is performed at every distinct frequency $\omega . \mathbf{U}(\omega)$ is an $n_{i} \times$ $n_{i}$ unitary matrix, $\mathbf{K}_{d d}(\omega)$ is an $n_{i} \times n_{i}$ full rank diagonal matrix with components $\kappa_{i}(\omega)>0$ which are the eigenvalues of $\boldsymbol{\Phi}_{d d}(\omega)$. Also perform the following eigendecomposition

$$
\begin{aligned}
& \mathbf{H}^{H}(\omega) \boldsymbol{\Phi}_{n n}^{-1}(\omega) \mathbf{H}(\omega)= \\
& {\left[\begin{array}{ll}
\mathbf{V}(\omega) & \overline{\mathbf{V}}(\omega)
\end{array}\right]\left[\begin{array}{cc}
\boldsymbol{\Lambda}_{n n}^{-1}(\omega) & \mathbf{0} \\
\mathbf{0} & \mathbf{0}
\end{array}\right]\left[\begin{array}{l}
\mathbf{V}^{H}(\omega) \\
\overline{\mathbf{V}}^{H}(\omega)
\end{array}\right]}
\end{aligned}
$$

where the $P \times P$ diagonal matrix $\boldsymbol{\Lambda}_{n n}^{-1}(\omega)$ contains the non-zero eigenvalues $\lambda_{i}^{-1}(\omega)$ of $\mathbf{H}^{H} \boldsymbol{\Phi}_{n n}^{-1} \mathbf{H}$ for a particular frequency $\omega$ and $P$ is defined as $P=\min \left\{n_{T}, n_{R}, \operatorname{rank}\{\mathbf{H}\}\right\}$. Without loss of generality $\lambda_{i}(\omega)$ are assumed to be sorted in ascending order, i.e. $0<\lambda_{1} \leq \lambda_{2} \leq \cdots \leq \lambda_{P}$. The $n_{T} \times P$ orthogonal matrix $\mathbf{V}(\omega)$ forms a basis for the range space of $\mathbf{H}^{H} \boldsymbol{\Phi}_{n n}^{-1} \mathbf{H}$ whereas the $n_{T} \times\left(n_{T}-P\right)$ orthogonal matrix $\overline{\mathbf{V}}(\omega)$ constitutes a basis for the null space. Also make the following two definitions on the transmitter filter and the TIR as

$$
\begin{aligned}
\mathbf{F}(\omega) & \triangleq \mathbf{V}^{H}(\omega) \mathbf{C}(\omega) \mathbf{U}(\omega) \\
\mathbf{B}_{d}(\omega) & \triangleq \mathbf{U}^{H}(\omega) \mathbf{B}(\omega) \mathbf{U}(\omega) .
\end{aligned}
$$

With the substitution of (10)-(13), theMSEin (9) can be expressed as

$$
J\left(\mathbf{F}, \mathbf{B}_{d}\right)=\operatorname{tr} \frac{1}{2 \pi} \int_{-\pi}^{\pi}\left\{\mathbf{B}_{d}\left[\mathbf{K}_{d d}^{-1}+\mathbf{F}^{H} \Lambda_{n n}^{-1} \mathbf{F}\right]^{-1} \mathbf{B}_{d}^{H}\right\} d \omega .
$$

Based on the results in [4], it can be proven that the structure of the optimum filters which minimize $J\left(\mathbf{F}, \mathbf{B}_{d}\right)$, i.e. $\mathbf{F}_{\text {opt }}(\omega)$ and $\mathbf{B}_{d, o p t}(\omega)$ are diagonal when $\kappa_{i}$, and the diagonal entries of $\mathbf{B}_{d, o p t}(\omega)$, i.e. $b_{d, i}(\omega), i=1, \ldots, n_{i}$ are ordered such that

$$
\kappa_{1}\left|b_{d, 1}\right|^{2} \geq \kappa_{2}\left|b_{d, 2}\right|^{2} \geq \cdots \geq \kappa_{n_{i}}\left|b_{d, n_{i}}\right|^{2} .
$$

A detailed proof can be found in [6]. With the diagonality of $\mathbf{F}_{\text {opt }}(\omega)$ and $\mathbf{B}_{\text {d,opt }}(\omega)$, (14) can be expressed as a set of independent SISO subsystems as follows

$$
J= \begin{cases}\frac{1}{2 \pi} \int_{-\pi}^{\pi} \sum_{i=1}^{n_{i}} \frac{\left|b_{d, i}\right|^{2} \kappa_{i} \lambda_{i}}{\lambda_{i}+\kappa_{i}\left|f_{i}\right|^{2}} d \omega & , n_{i} \leq P \\ \frac{1}{2 \pi} \int_{-\pi}^{\pi} \sum_{i=1}^{P} \frac{\left|b_{d, i}\right|^{2} \kappa_{i} \lambda_{i}}{\lambda_{i}+\kappa_{i}\left|f_{i}\right|^{2}} d \omega+\frac{1}{2 \pi} \int_{-\pi}^{\pi} \sum_{i=P+1}^{n_{i}}\left|b_{d, i}\right|^{2} \kappa_{i} d \omega, n_{i}>P\end{cases}
$$

where $f_{i}(\omega), i=1, \ldots, P$ are the diagonal elements of $\mathbf{F}_{\text {opt }}(\omega)$. In this paper, we consider only the case of $n_{i} \leq P$, and the case $n_{i}>P$ can be shown to be non-existent as in Remark 3. Also with the substitution of (10)-(13) into (6) and (7), the constraints can be written as

$$
\begin{aligned}
\sum_{i=1}^{n_{i}} \frac{1}{2 \pi} \int_{-\pi}^{\pi} \kappa_{i}\left|f_{i}\right|^{2} d \omega & \leq P_{o} \\
\frac{1}{2 \pi} \int_{-\pi}^{\pi}\left|b_{d, i}\right|^{2} d \omega & =1, \forall i .
\end{aligned}
$$

The design problem is now casted as finding the optimum $b_{d, i}(\omega)$ and $\left|f_{i}(\omega)\right|^{2}$ in (16) subject to (17) and (18) which appears to be not straightforward. However, realizing that (16) is 
$J(\mathbf{Q}(\omega), \mathbf{C}(\omega), \mathbf{B}(\omega))=\operatorname{tr} \frac{1}{2 \pi} \int_{-\pi}^{\pi}\left\{\left[e^{-j \omega m} \mathbf{B}(\omega)-\mathbf{Q}(\omega) \mathbf{H}(\omega) \mathbf{C}(\omega)\right] \mathbf{\Phi}_{d d}(\omega)\left[e^{-j \omega m} \mathbf{B}(\omega)-\mathbf{Q}(\omega) \mathbf{H}(\omega) \mathbf{C}(\omega)\right]^{H}+\mathbf{Q}(\omega) \mathbf{\Phi}_{n n}(\omega) \mathbf{Q}^{H}(\omega)\right\} d \omega$

jointly convex for the sets $\{\mathcal{B}\}=\left\{b_{d, i}, \forall i\right\}$ and $\{\mathcal{F}\}=\left\{\left|f_{i}\right|^{2}\right.$, $\forall i\}$, hence $J$ has a unique global minimum, an iterative approach is proposed, i.e. optimum $\left\{b_{d, i}\right\}$ is found by setting $\left\{\left|f_{i}\right|^{2}\right\}$ fixed and in the next round optimum $\left\{\left|f_{i}\right|^{2}\right\}$ is found by setting $\left\{b_{d, i}\right\}$ fixed. If this is repeated several times, the optimization converges to the vicinity of the global minimum. In fact, our simulations revealed that four iterations on average are adequate for convergence.

\section{TRANSMITTER FILTER OPTIMIZATION}

For a fixed set of $\left\{b_{d, i}(\omega)\right\}$, the optimum set of $\left\{\left|f_{i}(\omega)\right|^{2}\right\}$ for $J$ in (16) with the power constraint (17) is obtained using the Lagrange multiplier $1 / \gamma^{2}$ as

$$
\left|f_{i}(\omega)\right|^{2}=\max \left\{\gamma \sqrt{\left|b_{d, i}(\omega)\right|^{2} \frac{\lambda_{i}(\omega)}{\kappa_{i}(\omega)}}-\frac{\lambda_{i}(\omega)}{\kappa_{i}(\omega)}, 0\right\}
$$

$, i=1, \ldots, P$, for some waterlevel $\gamma$ and $\omega \in[0,2 \pi)$. The $\max \{\cdot, 0\}$ operator is used to avoid negative power levels. Dividing the frequency range into bins by $\omega_{l}=2 \pi l / N, l=0, \ldots, N-$ 1 , and defining $a_{k, l}=\sqrt{\left|b_{d, k}\left(\omega_{l}\right)\right|^{2} \lambda_{k}\left(\omega_{l}\right) / \kappa_{k}\left(\omega_{l}\right)}$ and $b_{k, l}=$ $\lambda_{k}\left(\omega_{l}\right) / \kappa_{k}\left(\omega_{l}\right)$, this expression can be put in an algorithm form as an extension of [5] as follows

1. Let the set of ordered pairs $(k, l) \forall k, l$ are sorted in ascending order according to the quotients $q(j)=a_{k, l} / b_{k, l}$, $k=1, \ldots, P, l=0, \ldots, N-1$ and $j=l P+k=1, \ldots, N P$ yielding an $N P \times 1$ vector $\mathcal{S}$,

2. Calculate the waterlevel $\gamma$ according to

$$
\gamma=\left(P_{o}+\sum_{j \in \mathcal{S}} a_{k, l}\right) / \sum_{j \in \mathcal{S}} b_{k, l},
$$

3. If the last element of the set $\mathcal{S}$ accepts a negative value, i.e. $\left|f_{k}\left(\omega_{l}\right)\right|^{2}=\gamma b_{k, l}-a_{k, l}<0$ then remove the pair $(k, l)$ from $\mathcal{S}$ and move the cursor to one upper position and go to Step 2,

4. If no negative value is left, stop the loop and calculate the final power levels for each bin according to (19) using the waterlevel obtained from the last step.

This algorithm finds the optimum transmitter filter coefficients in at most $N P$ loops.

\section{TIR OPTIMIZATION}

Now, keeping the transmitter filter $\mathbf{F}$ fixed, we compute the optimum TIR coefficients. Similar to the definition of TIR in (1) define the transformed TIR for each subsystem as follows

$$
\begin{aligned}
b_{d, i}(\omega) & =b_{d, i, 0}+b_{d, i, 1} e^{-j \omega}+\cdots+b_{d, i, n_{B^{-}}} e^{-j \omega\left(n_{B^{-}}-1\right)} \\
& =\mathbf{b}_{d, i}^{T} \mathbf{w}
\end{aligned}
$$

for all $i, \mathbf{b}_{d, i}=\left[\begin{array}{llll}b_{d, i, 0} & b_{d, i, 1} & \cdots & b_{d, i, n_{B}-1}\end{array}\right]^{T}$ and $\mathbf{w}=\left[\begin{array}{ll}1 e^{-j \omega} & 0\end{array}\right.$ $\left.\cdots e^{-j \omega\left(n_{B}-1\right)}\right]^{T}$. Substitution of (21) into (16) and (18) yields

$$
\begin{gathered}
\text { minimize } J_{i}=\mathbf{b}_{d, i}^{H} \mathbf{R}_{i} \mathbf{b}_{d, i} \\
\text { subject to } \mathbf{b}_{d, i}^{H} \mathbf{b}_{d, i}=1
\end{gathered}
$$

where

$$
\begin{aligned}
\mathbf{R}_{i} & =\frac{1}{2 \pi} \int_{-\pi}^{\pi} \mathbf{w}^{*} \frac{\kappa_{i} \lambda_{i}}{\lambda_{i}+\kappa_{i}\left|f_{i}\right|^{2}} \mathbf{w}^{T} d \omega \\
{\left[\mathbf{R}_{i}\right]_{k, l} } & =\frac{1}{2 \pi} \int_{-\pi}^{\pi} \frac{\kappa_{i} \lambda_{i}}{\lambda_{i}+\kappa_{i}\left|f_{i}\right|^{2}} e^{j \omega(k-l)} d \omega .
\end{aligned}
$$

However, observe that (25) is the inverse Fourier transform of the spectral density function $\kappa_{i} \lambda_{i} /\left(\lambda_{i}+\kappa_{i}\left|f_{i}\right|^{2}\right)$, hence it is an autocorrelation matrix. Therefore, the minimization in (22)-(23) becomes the standard eigenvalue problem, and the solution for $\mathbf{b}_{d, i}$ is the eigenvector corresponding to the smallest eigenvalue of $\mathbf{R}_{i}$. Repeating this procedure for all $n_{i}$ subsystems, one can find the optimum TIR for the MIMO system by substituting $b_{d, i}(\omega)$ into $\mathbf{B}_{d}(\omega)$.

\section{REMARKS}

1. The issue of the starting point of the iteration of finding the optimum transmitter filter and TIR is not important since it can be proven that (16) is convex for all $b_{d, i}(\omega) \in \mathbb{C}$ and $\left|f_{i}(\omega)\right|^{2} \in \mathbb{R}_{0+}$ [6], hence $\mathbf{B}_{d}=\mathbf{F}=\mathbf{I}$ is a logical initialization.

2. From (19) it is observed that the phase of $f_{k}$ is not important. Hence, the phase of $f_{k}$ does not affect the corresponding target impulse response design in the subsequent step.

3. In (16), $n_{i}>P$ means the number of input streams is greater than the available eigenmodes. However, only $P$ streams can be transmitted from these eigenmodes, and $n_{i}-P$ streams will be lost. Since the transmitter knows the CSI, hence $P$, it must limit the number of input stream to $P$. Therefore, practically the case $n_{i}>P$ should not exist.

4. From (8) and (9) it can be seen that the $e^{-j \omega m}$ term only appears as a delay in the optimum receiver filter design and does $n o t$ affect the minimization of the cost function $J(\mathbf{C}, \mathbf{B})$. This property, which was also reported by Martin, et al in [7], can be explained as follows. When the filter length is finite, the error autocorrelation matrix, from which the TIR is calculated, is Hermitian symmetric, but not Toeplitz. This can be seen using the notation of [3], i.e. $\mathbf{R}_{e e}=\left[\mathbf{R}_{x x}^{-1}+\mathbf{H}^{H} \mathbf{R}_{n n}^{-1} \mathbf{H}\right]^{-1}$ where $\mathbf{R}_{e e}, \mathbf{R}_{x x}$ and $\mathbf{R}_{n n}$ are the autocorrelation matrices of error, input and noise. The term $\mathbf{H}$ in this case is the convolution matrix composed of the channel coefficients. Due to the truncation at the edges of this convolution matrix, the positions close to the edges of the autocorrelation matrix $\mathbf{R}_{e e}$ does not provide the actual autocorrelation function. It is reported in [3] that the optimum value of the delay parameter tends to be in the center of $\mathbf{R}_{e e}$, which is more closer to the actual autocorrelation function compared to the edges. As the length of the filter increases, $\mathbf{R}_{e e}$ tends to be Toeplitz, and all delays provide the same performance. In fact, in our case the spectral density function $\left[\boldsymbol{\Phi}_{d d}^{-1}+\mathbf{C}^{H} \mathbf{H}^{H} \boldsymbol{\Phi}_{n n}^{-1} \mathbf{H C}\right]^{-1}$ is the Fourier transform of such a Toeplitz error autocorrelation matrix. Hence, the optimization is insensitive to the delay $m$.

\section{SIMULATIONS AND RESULTS}

One of the measure of performance adopted in this study is the shortening signal-to-interference-plus-noise ratio (SINR) at the out- 


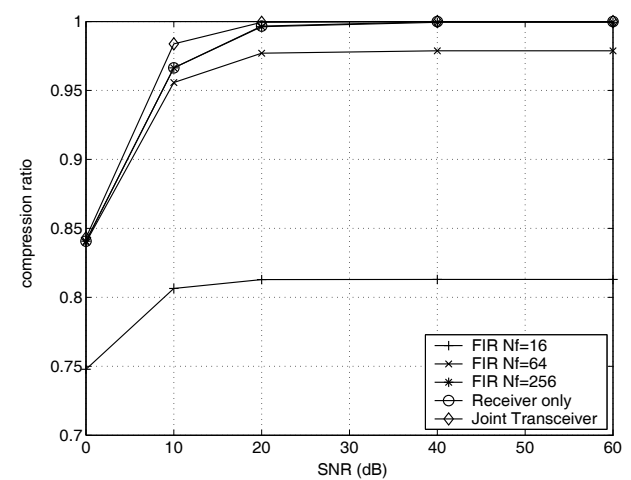

Fig. 2. Compression ratio comparison of the finite length time domain design for filter lengths, 16, 64 and 256 to the proposed frequency domain designs with both receiver-only and joint transceiver processing.

put of the channel shortening filter defined as the ratio of the energy of the equalized channel contained in the window determined by the TIR to the out-of-window energy plus noise power. Another related performance measure is the compression ratio defined as the ratio of the total energy that remains in the window after shortening, to the total energy in the entire equalized channel impulse response.

For comparison reason, the performance of the proposed frequency-domain method is also evaluated for the receiver-only case by setting $\mathbf{C}=\mathbf{I}$. The performance of the proposed frequency domain approach for both receiver-only and joint transceiver is compared to that of the finite length time domain MMSE scheme of [3] with filter lengths 16,64 and 256. A 16 tap $2 \times 2$ MIMO channel is shortened to a 2 tap equalized one. For the joint transceiver design, the number of iterations is ten, however, it is observed that most of the time the optimum is attained within 3-4 iterations. Also the frequency range $[0,2 \pi)$ is divided into 256 bins.

Figure 2 provides the compression ratio comparison of the proposed frequency domain method for both joint transceiver and receiver-only cases and for the FIR channel shortening filters. It is observed that the joint transceiver scheme outperforms the frequency domain receiver-only design in low to medium SNR region. This is due to the extra degree of freedom that the transmitter has in order to mitigate the channel noise. On the other hand, the performance of the FIR design (except for very long lengths) is worse than that of the proposed frequency domain designs and experiences a floor in performance due to the incapability of equalizing the channel with finite length.

Consistent with the result in Figure 2, the SINR curve has similar behaviour as seen in Figure 3. In this case the noise power is also taken into account. It is seen that while there is a flooring effect in the FIR filter performance, the proposed method has a linear curve. Except very long FIR filter, the frequency domain approach achieves much better performance. Moreover, the gain obtained by employing joint transceiver algorithm is $3 \mathrm{~dB}$ better than that of the frequency domain receiver-only design at high SNR.

\section{CONCLUSIONS}

In this paper, we proposed a joint transmitter and receiver design for the MMSE MIMO channel shortening filter problem. It is

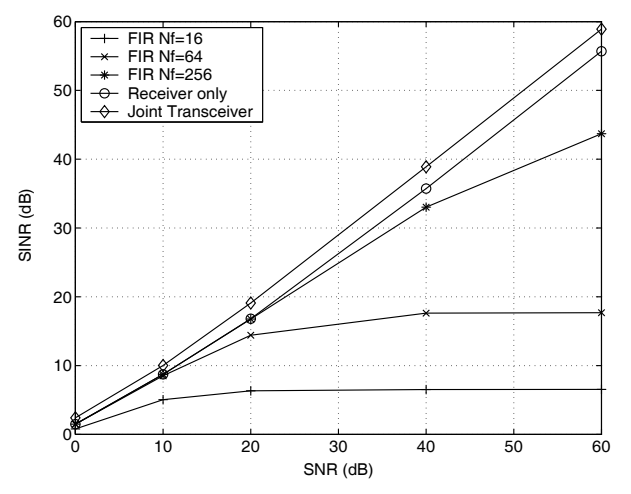

Fig. 3. SINR comparison of the finite length time domain design for filter lengths, 16, 64 and 256 to the proposed frequency domain designs with both receiver-only and joint transceiver processing.

demonstrated that with proper transformations, the problem can be expressed using a set of independent SISO subsystems. Since a frequency domain approach is equivalent to infinite length filters, the performance of the proposed method is much better than that of an FIR channel shortening filter. Due to the extra degree of freedom that the transmitter filter possesses, the noise amplification is reduced and the compression ratio is enhanced. Hence, the proposed method is found to be suitable for scenarios where CSI is available at the transmitter.

\section{REFERENCES}

[1] D. D. Falconer and F. R. Magee, "Adaptive channel memory truncation for maximum likelihood sequence estimation," Bell Sys. Tech. Journal, pp. 1541-1562, Nov. 1973.

[2] R. K. Martin, K. Vanbleu, M. Ding, G. Ysebaert, M. Milosevic, B. L. Evans, M. Moonen, C. R. Johnson, Jr., "Unification and evaluation of equalization structures and design algorithms for discrete multitone modulation systems," accepted for publication in IEEE Trans. Signal Processing

[3] N. Al-Dhahir, "FIR channel shortening equalizers for MIMO ISI channels," IEEE Trans. Commun., vol. 49, no. 2, pp. 213218, Feb. 2001.

[4] J. Yang and S. Roy, "On joint transmitter and receiver optimization for multiple-input-multiple-output (MIMO) transmission systems," IEEE Trans. Commun., vol. 42, no. 12, pp. 3221-3231, Dec. 1994.

[5] D. P. Palomar and M. A. Lagunas, "Joint transmit-receive space-time equalization in spatially correlated MIMO channels: A beamforming approach," IEEE J. Select. Areas Commun., vol. 21, no. 5, pp. 730-743, June 2003.

[6] C. Toker, Signal Processing Algorithms and Architectures for Communication Transceivers, PhD dissertation, Department of Electronic Engineering, King's College, University of London, UK., Nov. 2004.

[7] R. K. Martin, M. Ding, B. L. Evans and C. R. Johnson, Jr., "Infinite length results and design impliciations for time-domain equalizers," IEEE Trans. Signal Processing, vol. 52, no. 1, pp. 297-301, Jan. 2004. 